\title{
Elderly People Sit to Stand Transfer Experimental Analysis
}

\author{
P. Médéric, V. Pasqui, F. Plumet and P. Bidaud \\ Laboratoire de Robotique de Paris (LRP) \\ CNRS FRE 2507 - Université Pierre et Marie Curie, Paris 6 \\ 18, route du Panorama, BP 61, 92265 Fontenay-aux-Roses, France
}

\begin{abstract}
This paper describe an assistive device for the elderly providing support during the sit to stand transfer. A postural stabilization criteria and an assisting force in case of instability are presented.
\end{abstract}

Keywords: assisting device, transfer assistance, stability criteria

\section{Introduction}

The elderly population is growing likewise numbers of resident in assisted living facilities. Medical staff are more busy with daily living tasks such as escorting the patient or assist them during transfer. Due to the disparity between the member of the medical staff and the elderly, a lot of patient are not assisted and stayed in an inactivity leading to the loss of the walking function and mortality [1].

Moreover many nursing home workers are suffering of low back injuries caused by the high frequency of patient lifting [2],[3]. On the one hand conventional walker have been developed to provide support during the walk on the other hand patient lifter have been use concerning the transfer of the patient. However these kind of device are not totally useful for all patient, pathologies and medical staff [4], various institutes started the development of intelligent robot.

The Pam-Aid [5] and the Pamm projects with the SmartCane [6] and the smart walker [7] provide support during the walk and allow obstacles avoidance and path navigation. The Care-O-Bot [8],[9], and the Nursebot 
[10], are assistive devices for the daily life. The Care-O-Bot is equipped with a manipulator arm to catch object, the movement of its platform is deal with sensors on the handles and a path navigation system, it also provide information to the user like taking medicine.

The Nursebot, which do not afford a physical support, is studied for the users having failing memory and disorientation. The goal of this project is to augment the human interaction by reminding people about important routine activities and guiding them with a path navigation system.

All the previous works raise the problems of path navigation with obstacle avoidance and shared control. Relating to the transfer assistance of the elderly a power-assistance device has been developed by the Ritsumeikan university [11], it ensure a physical support during the walk and the sit to stand transfer. The entire system have to be in a defined room, the trajectory and the supporting forces do not evolve in accordance with the configuration of the patient and this kind of assistive device can not be use outside a defined room. An assistive device (AD) have been developed in our laboratory. We emphasis on a device combined the transfer and the walk. This device provide support during the walk and also during the sit to stand transfer, it is primarily intended for elderly patients who have falling background [12]. We focus on postural stability especially during the verticalisation, postural observation and estimation is implemented on the assistive device in order to monitor the patient and modify its posture.

This paper presents experimental results done with elderly and a postural stabilization criteria during the transfer.

\section{System overview}

The assistive device (AD) is a two degree of freedom mechanism mounted on an active mobile platform (see fig. 1). During the transfer assistance the handles of the AD pull slowly the patient to the up position. This is obtained by using two parallel and independent mechanisms combined in a serial way.

The trajectory of the handles relay on a trajectory generator, it allow the patient to get off its retropulsion configuration to an antepulsion configuration. Force sensors are added on each handle to measure force interaction between the user and the device. Details on the design the $\mathrm{AD}$ and its trajectory generator can be found in [12]. 


\section{DRAFT}

Elderly People Sit to Stand Transfer Experimental Analysis
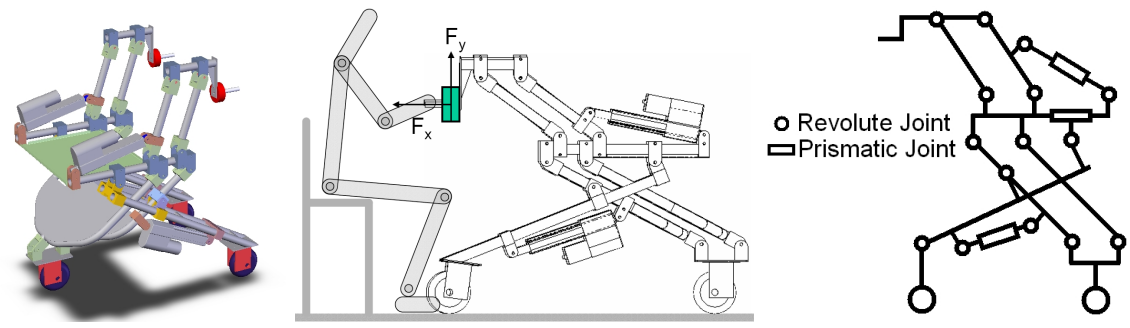

Fig. 1. Description of the assistive device

\section{Transfer assistance for elderly}

In order to demonstrate the efficiency of our $\mathrm{AD}$ during the transfer, several experimental standing-up and siting-down trials were executed. Measurement series has been done on a set of 19 elderly patients in Charles-Foix Hospital. The patients are affected with different pathologies disturbing the transfer. The device is used to provide support during the transfer, gerontologists choose, according to the patient, the position of the initial and final points of the assistive trajectory. During the movement the patient hold the handles of the prototype, force measurement are done on each handles. On the 19 patients only 2 could not stand up with the help of the $\mathrm{AD}$, these two patients have serious impairment (acute hemiplegia) that did not allow us to stand up without the assistance of member of the medical staff.

Obviously, patient who could not stand up with an external help could not use the device to stand up. Patient who could stand up without a help could stand up thanks to the AD.

Due to their physical condition and age the patient could not produce many trial, nevertheless concerning the force measurement we can observe a repeatability of the measurement on each user. The global shape is the same for each trials of the same patient but it is not the same between the patients.

Concerning the sit to stand transfer the effort along the $\mathbf{y}$ axis (fig. 1) do not evolve in the same way, see fig. 2. For the patient 1 its seems that the patient lift up the right handle $(\mathbf{F y}>0)$ whereas for the patient 2 (if we do not consider the first trial $\mathbf{F y} \approx 0$ ) the force interaction $\mathbf{F y}$ is still evolve in the negative value meaning that the patient is still pushing during the transfer with a maximum force $\mathbf{F y}=-140 \mathrm{~N}$ around $19 \%$ of his weight. The opposite direction of the effort $\mathbf{F y}$ between the left and the right handle indicate that the patient 1 is tipping over on his left side. 

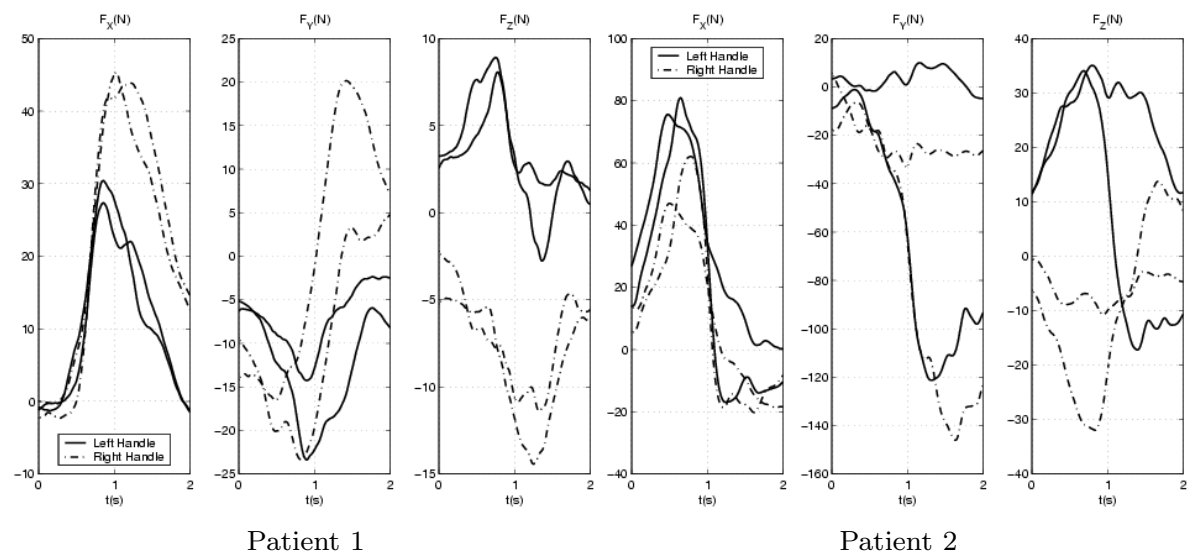

Fig. 2. Force interaction during sit to stand transfer

During the stand to sit transfer we observe the same phenomena of opposite effort $(\mathbf{F y})$ for the patient 1, see fig. 3. The analysis of the stand to sit transfer is more difficult because most of the patient let their body fall back to the chair. The interpretation of the effort is getting more complex, the measurement of the force interaction between the patient and the chair could be useful for a complete identification of the transfer.
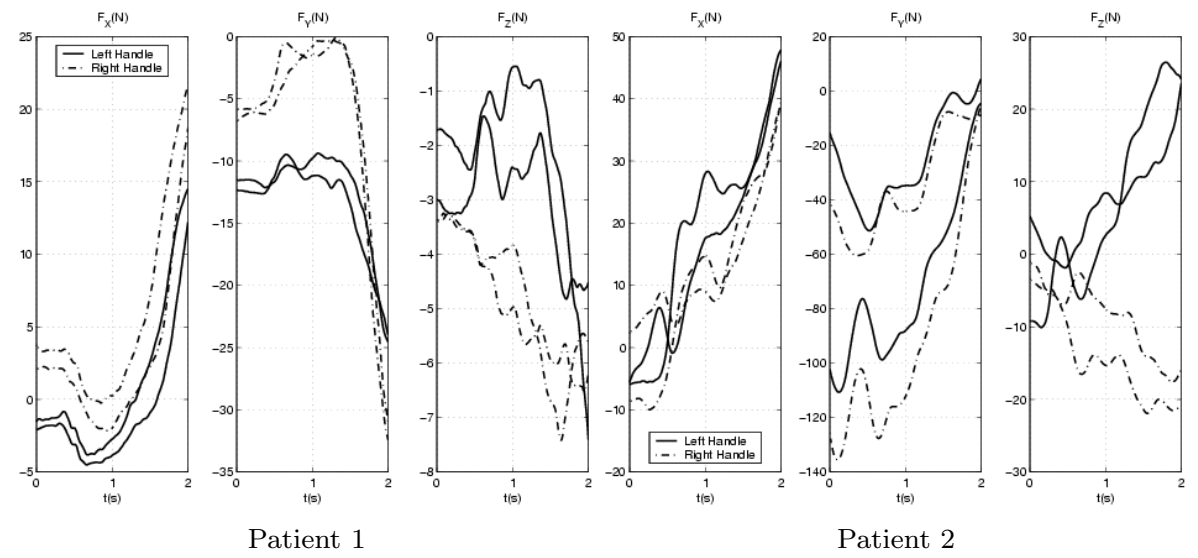

Fig. 3. Force interaction during stand to sit transfer

Theses previous interpretations do not allowed us to identify the posture of the patient during the transfer. Observations of the force interaction between the patient, the chair and also the floor added with measurement 


\section{DRAFT}

Elderly People Sit to Stand Transfer Experimental Analysis

of motion of the body segment could give an interesting observation of the patient's posture (see fig. 4).

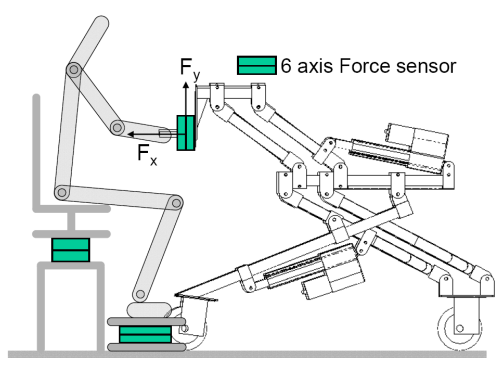

Fig. 4. Future test bench

In order to compensate the fall during the transfer we design a criteria of stability, next section will present this criteria.

\section{Stability criteria}

Compensation of the fall during the transfer relay on a stability criteria, the $\mathrm{AD}$ have to produce the trajectory and force compensation helpful for the user in case of instability. In the following sub section we will introduce a stability criteria of the patient.

\subsection{Design of a stability criteria}

This stability criteria is based on the establishment of the Zero Moment Point (ZMP) [13] on a simplified model of the patient.

The patient is a 7 bar mechanism studied in the sagittal plane $\left(x_{o}, y_{o}\right)$ (see fig. 5), $m_{i}$ is the mass of the $i^{t h}$ segment, $M$ is the total mass of the patient, $X_{z m p}$ is the position of the ZMP along the $\mathbf{x}_{\mathbf{o}}$ axis in the frame $R_{0}$.

Using the Newton-Euler formulation at the ZMP we get the equation of the position of the ZMP during the transfer:

$$
X_{z m p}=\frac{H_{Z}+M\left(\ddot{Y}_{G} X_{G}-\ddot{X}_{G} Y_{G}\right)+\sum_{i=1}^{i=n} m_{i} g X_{G i}}{M\left(\ddot{Y}_{G}+g\right)}
$$




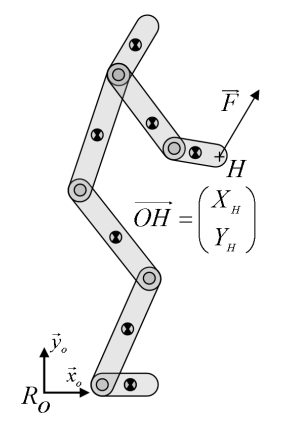

Fig. 5. Simplified model

By considering the effect of inertia of each segment lower in front of the inertia of the trunk, the expression of the ZMP become:

$$
X_{z m p}=\frac{\ddot{Y}_{G} X_{G}-\ddot{X}_{G} Y_{G}+g X_{G}}{\ddot{Y}_{G}+g}
$$

The stability criteria of the patient is done by using the ZMP equation, the patient posture is defined stable if $X_{z m p}$ is included in the foot's area. In case of instability the $\mathrm{AD}$ had to produce the assisting force to remain the ZMP into the foot's area. In the next sub section will present the assisting force model.

\subsection{Assisting force model}

The goal of the assisting force is to ensure the postural stability of the patient. The force interaction $\mathbf{F}$ between the patient and the AD is defined into the hand position of the patient at the point $H$ (see fig. 5). By included $\mathbf{F}$ into the Newton Euler formulation (eq. 1) we get an other expression of the $X_{z m p}$ :

$$
X_{z m p}=\frac{M\left(\ddot{Y}_{G}+g\right) X_{G}-M \ddot{X}_{G} Y_{G}-F_{X} Y_{H}+F_{Y} X_{H}}{M\left(g+\ddot{Y}_{G}\right)-F_{Y}}
$$

With the stability criteria :

$$
a \leq X_{z m p} \leq b
$$

According to eqs. 3 and 4 if the patient is in an instable posture the $\mathrm{AD}$ had to provide the assisting force to remains the patient into a safe posture. To solve this problem we express the force interaction $\mathbf{F}$ under 
a non linear problem under constrained, by finding the minimal effort $\mathbf{F}$ to the following formulation:

Minimize $F=\sqrt{F_{X}^{2}+F_{Y}^{2}}$ subject to:

$$
\left[\begin{array}{cc}
Y_{H} & -\left(X_{H}+a\right) \\
-Y_{H} & \left(b+X_{H}\right)
\end{array}\right]\left[\begin{array}{c}
F_{X} \\
F_{Y}
\end{array}\right] \leq\left[\begin{array}{c}
M\left(\left(g+\ddot{Y}_{G}\right)\left(X_{G}-a\right)-\ddot{X}_{G} Y_{G}\right) \\
M\left(\left(g+\ddot{Y}_{G}\right)\left(b-X_{G}\right)+\ddot{X}_{G} Y_{G}\right)
\end{array}\right]
$$

With this model of assisting force we need kinetics information of the patient. A new test platform added with motion sensors on the patient will permit us to validate this model.

\section{Conclusion}

An assisting device for elderly patient has been developed. First experiments with elderly have been done on the transfer function. These first experiments showed the efficiency of the assisting device, only 2 out of 19 patients could not stand up with it. With its sensors the assisting device can provide information on the posture of the patient and also providing support to the user in case of instability. This device can also be a tool for the kinesitherapist, it can give a powerful programmable device for the monitoring and rehabilitation of the lower limbs. A stability criteria and an assisting force models have been done to observe posture and to stabilize the patient during the transfer. Experimentation and validation of these models will be done soon.

\section{Acknowledgments}

This work is partly supported by the French RNTS (Réseau National des Technologies de la Santé) program under grant N $02 B 0414$ (Monimad Project).

\section{References}

1. M. Hirvensalo, T. Rantanen, and E. Heikkinen. Mobility difficulties and physical activity as predictors of mortality and loss of independence in the community-living older population. Journal of the American Geriatric Society, 48:493-498, 2000. 
2. T.J. Stobbe, R.W. Plummer, R.C. Jensen, and M.D. Attfield. Incidence of low back injuries among nursing personnel as a function of patient lifting frequency. Journal of Safety Research, 19(1):21-28, Spring 1988.

3. Z. Zhuang, T. J. Stobbe, H. Hsiao, J. W. Collins, and G. R. Hobbs. Biomechanical evaluation of assistive devices for transferring residents. Applied Ergonomics, 30(4):285-294, August 1999.

4. S. Ruszala and I. Musa. An evaluation of equipment to assist patient sit-tostand activities in physiotherapy. Physiotherapy, 91(1):35-41, March 2005.

5. G. Lacey, S. Mac Namara, and K. M. Dawson-Howe. Personal adaptive mobility aid for the infirm and elderly blind. Lecture Notes in Computer Science, 1458:211-220, 1998.

6. S. Dubowsky, F. Genot, S. Godding, H. Kozono, A. Skwersky, H. Yu, and L.S. Yu. Pamm - a robotic aid to the elderly for mobility assistance and monitoring: A "helping-hand" for the elderly. In IEEE International Conference on Robotics and Automation, volume 1, pages 570-576, San Francisco, USA, 2000 .

7. H. Yu, M. Spenko, and S. Dubowsky. An adaptive shared control system for an intelligent mobility aid for the elderly. Autonomous Robots, 15:53-66, 2003.

8. R.D. Schraft, C. Schaeffer, and T. May. Care-O-bot ${ }^{\mathrm{TM}}$ : The concept of a system for assisting elderly or disabled persons in home environments. In Proceedings of the 24th Annual Conference of the IEEE Industrial Electronics Society: IECON 98, volume 14, pages 2476-2481, Aachen, Germany, 1998.

9. B. Graf, M. Hans, and D. S. Rolf. Care-O-bot II - development of a next generation robotic home assistant. Autonomous Robots, 16:193-205, 2004.

10. G. et al. Baltus. Towards personal service robots for the elderly. In Proceeding of the 2000 Workshop on Interactive Robotics and Entertainment (WIRE-2000), Pittsburgh,USA, 2000.

11. K. Nagai, I. Nakanishi, and H. Hanafusa. Assistance of self-transfer of patients using a power-assisting device. In IEEE International Conference on Robotics and Automation, pages 4008-4015, Taipei, Taiwan, 2003.

12. P. Médéric, V. Pasqui, F. Plumet, P. Bidaud, and J.C. Guinot. Design of a walking-aid and sit-to-stand transfer assisting device for elderly people. In 7th Int. Conference on Climbing on Walking Robots (CLAWAR'04), Madrid, Spain, 2004.

13. M. Vukobratovic and B. Borovac. Zero moment point thirthy five years of its life. International Journal of Humanoid Robotics, 1(1):157-173, 2004. 